\title{
The expanding earth and formation of satellites
}

\begin{abstract}
The gravity holds us on surface of Earth according to Newton this statement was true but this paper will show a totally different view. The universe is not in the same state as it was formed it is true for the Earth also, our Earth is expanding since inception and this paper will prove the statement right not only this paper meant to show how and when exactly a satellite like moon will form. The gravity holds us on surface of Earth according to Newton this statement was true but this paper will show a totally different view. The universe is not in the same state as it was formed it is true for the Earth also, our Earth is expanding since inception and this paper will prove the statement right not only this paper meant to show how and when exactly a satellite like moon will form.
\end{abstract}

Volume 3 Issue $3-2019$

\author{
Mayank Yadav, Kshitiz Mittal \\ Department of physics, University Rajasthan College, India
}

Correspondence: Mayank Yadav, Department of physics, University Rajasthan College, 24, shekhawati nagar, vki I4, Jaipur, Rajasthan, India (302013), India, Tel 8890849682,

Emailm.yadav1238@gmail.com

Received: May 24, 2019| Published: June 24, 2019

Keywords: planet, moon, earth, satellite, celestial bodies, equator curve, theories on mountain

\section{Formation of planet}

A planet is a part of the parent star that is it has the same composition as the star but the question is how it forms.

Let it be star of M-mass, $r$ - Radius, W-angular velocity and three points on surface of star at equator, at 45 degree angle, at pole.

I. Point on equator: This point is experiencing two forces i.e. centripetal force and centrifugal force.

\section{Centrifugal force- $M r W 2$}

Gravity we feel $=$ Centripetal force $-M r W 2$

That shows gravity told by Newton is actually a resultant not the force itself so the force itself would be

Force $=$ Gravity we feel $+M r W 2$

And the point will feel 0 gravity when

$\rightarrow \mathrm{MrW} 2$ = centripetal force

After this point bulge on equator will likely to form that can be result into planet formation.

II. Point at 45 degree and pole has just opposite scenarios where velocity is not much to provide centrifugal force and revolution but they will simply escape after explosion.

The question here arises why this explosion is happening this can be simply understood by the plate formation.

Let us take an example of melted iron it is in liquid form but after sometime its upper layer solidifies but beneath it is still in liquid, same like this sun was hot initially and subsequently cooled with its upper layer being solidified, then due to sharp temperature gradient it exploded. Planets cooled down slowly and became what they are now. Let us take the example of moon for satellite. Moon is tidally locked with Earth i.e. revolves and rotates with same speed i.e. 27.322 days. If moon was part of Earth - Velocity at the time of escape will be velocity now as there is no drag. So $\mathrm{V} 0=\mathrm{V} 1$

$M r W 2=M g$ To escape from earth;

$r W 2=g(9.780 \mathrm{~m} / \mathrm{h})$
Velocity at the time of escape will be velocity now as there is no drag. So $\mathrm{V} 0=\mathrm{NOW}$

$$
\mathrm{V} 0=2 \times 3.14 \times 384400 \div 27.322 \times 24 \text {. }
$$

$\mathrm{V} 0=3681.45$

That is quite high rotational speed at the time earth was in formative stage and its upper layer was still soft and tear apart as to form moon, and if we look a closer look at the current speed of rotation of Earth i.e.

$$
\begin{aligned}
& \mathrm{V} 1=2 \times 3.14 \times 6400 \div 24 \\
& \mathrm{~V} 1=1674
\end{aligned}
$$

That is quite simple to understand that the rotational speed has gone decrease and as the system is closed one, we can conclusively say that Earth has gone its angular momentum decrease. We can conclude that radius of Earth has changed and it has enlarged. Though it is quite abnormal to take the idea of expanding Earth and other celestial bodies, but it is quite normal in that large force acting on mass. The shape of object or celestial body changes dramatically from almost a flat top ball with great bulge at equator curve like parabola then it break to form satellite or planets from it and shape of body comes back to Earth like shape. Let us look what would happen when a body goes with this change how its surface structure feels the change.

\section{Effect of expansion}

If we take a look on our Earth it is $27 \%$ of water over surface in the form of Oceans mainly, what if this was not always there, that is true water was not of our surface part earlier but it was a component present inside earth in form of solid water, gases, mixture with rocks and other forms. It got out from inside because of vents from which lava erupted with the water in form of gases and through rains it filled up into the gaps created by vents in process of millions of years. The vents got enlarged because the rotational speed that was $3681 \mathrm{~km} / \mathrm{h}$ and more and more water come over and over again from mid oceanic ridges. These ridges can be seen all over the world in almost all the seas, these ridges matches with the exact time when it got started. When Harry Hess propounded his Sea Surface Spreading theory he researched and found that time period of the rocks equidistance from mid oceanic ridges are same and there magnetic properties are same. 
That shows that they moved apart. So if that happened that should be the same result of the result we found in upper part. If sea was not present surface area now would be $27 \%$ more than at time there was no sea.

$$
4 \pi \mathrm{R} 2=1.27(4 \pi(\mathrm{R} 0) 2)
$$

$$
\mathrm{R} 0=5679.018
$$

That shows how our Earth has changed from $5679.018 \mathrm{~km}$ to $6400 \mathrm{~km}$ in radius from the time it started its journey. There the theory also discards the early theories on mountain and ocean formation such as Continental Drift Theory and Plate Tectonic Theory, but theory respects the early propounded theories as they were earliest to give this field new thought.

\section{Expanding earth theory and mountain formation}

There we can understand by this that our Earth has gone with a significant changes and mountains are one of them, to understand it there is one phenomenon called differential expansion, as the Earth has expanded it was not in uniform way but there was weak points which erupted more lava than others thus forming larger mid oceanic ridges. As we know the Pacific mid oceanic ridges that is around 24$33 \mathrm{~mm} /$ year are faster than the Atlantic one around $20 \mathrm{~mm} /$ year, so the movement Pacific mid oceanic ridges created is more than created by Atlantic Ocean. So the western side of United States got folded under the compressive forces of differential expansion.

\section{Conclusion}

There is always a change and change is inevitable in the universe this eternal change can be understood only in larger perspective, this theory gives idea about formation of planets in the equatorial line and briefs about formation of Moon. It touched the aspect of mountain formation by theory of Expanding Earth, there is always a lot to discover and a lot to share till the world be open to adapt.

\section{Acknowledgments}

None.

\section{Conflicts of interest}

The author declares there is no conflict of interest. 